\title{
CpG-depleted adeno-associated virus vectors evade immune detection
}

\author{
Susan M. Faust, ${ }^{1}$ Peter Bell,, Benjamin J. Cutler, ${ }^{1}$ Scott N. Ashley, ${ }^{1}$ Yanqing Zhu, ${ }^{1}$ \\ Joseph E. Rabinowitz, ${ }^{2}$ and James M. Wilson ${ }^{1}$
}

\begin{abstract}
${ }^{1}$ Gene Therapy Program, Department of Pathology and Laboratory Medicine, Perelman School of Medicine, University of Pennsylvania, Philadelphia, Pennsylvania, USA. ${ }^{2}$ Temple University, Department of Pharmacology, Philadelphia, Pennsylvania, USA.
\end{abstract}

\begin{abstract}
Due to their efficient transduction potential, adeno-associated virus (AAV) vectors are leading candidates for gene therapy in skeletal muscle diseases. However, immune responses toward the vector or transgene product have been observed in preclinical and clinical studies. TLR9 has been implicated in promoting AAV-directed immune responses, but vectors have not been developed to circumvent this barrier. To assess the requirement of TLR9 in promoting immunity toward AAV-associated antigens following skeletal muscle gene transfer in mice, we compared immunological responses in WT and Tlr9-deficient mice that received an AAV vector with an immunogenic capsid, AAVrh32.33. In Tlr9-deficient mice, IFN- $\gamma$ T cell responses toward capsid and transgene antigen were suppressed, resulting in minimal cellular infiltrate and stable transgene expression in target muscles. These findings suggest that AAV-directed immune responses may be circumvented by depleting the ligand for TLR9 (CpG sequences) from the vector genome. Indeed, we found that CpG-depleted AAVrh32.33 vectors could establish persistent transgene expression, evade immunity, and minimize infiltration of effector cells. Thus, $\mathrm{CPG}$-depleted $\mathrm{AAV}$ vectors could improve outcome of clinical trials of gene therapy for skeletal muscle disease.
\end{abstract}

\section{Introduction}

Muscle-directed gene delivery of structural proteins, such as sarcoglycans and dystrophin, offers a promising therapy for patients affected with muscular dystrophy. Adeno-associated virus (AAV) vector efficiently transduces dividing or nondividing muscle cells through both local and systemic administration, providing multiple strategies to treat musculoskeletal diseases. While thought to be minimally immunogenic - an attribute that allows for sustained transgene expression from $A A V$ vectors in some experimental models - adaptive immune responses have been observed and, in some cases, implicated in the loss of transgene expression (1-6).

Potential immune toxicity of AAV is complex and multifactorial (7). Activation of de novo and preexisting cellular and humoral immunoreactivity toward viral capsid and encoded transgene antigens has the potential to limit long-term transgene expression. TLRs recognize an array of pathogen-associated molecular patterns (PAMPs) of infectious microbes and are critically important for the induction of proinflammatory immune responses that initiate innate and adaptive immune responses toward the foreign pathogen (8). TLRs are highly expressed on innate immune cells such as macrophages and mast cells, dendritic cells, and to a lesser extent, B and T cells, endothelial cells, epithelial cells, and fibroblasts. TLR9 senses the major pathogenic DNA viruses including herpes simplex virus (HSV), EBV, CMV, adenovirus, and human papilloma virus $(9,10)$. TLR9 is found almost exclusively within intracellular compartments such as endosomes (11). This receptor is capable of recognizing unmethylated bacterial and viral $\mathrm{CPG}$ motifs initiating signaling pathways that activate the transcription factors NF-KB, IFN regulatory factors (IRFs), and activating

Conflict of interest: J.M. Wilson is a founder of and consultant to ReGenX and is an inventor on patents licensed to ReGenX and its affiliates. J.E. Rabinowitz, S.M. Faust, and J.M. Wilson are inventors on patents related to AAV CPG-depletion technology. Citation for this article: J Clin Invest. 2013;123(7):2994-3001. doi:10.1172/JCI68205. protein-1 (AP-1). TLR9 signaling can lead to the production of inflammatory cytokines IL-6, TNF- $\alpha$, type I IFNs, and IL-1 $\beta$, initiating both innate and adaptive immune responses (12). In addition, $\mathrm{CPG}$ motifs have been demonstrated to induce chemokines, MHC II molecules, and costimulatory ligands on myocytes, which have been postulated to act as nonprofessional $\operatorname{APCs}(13,14)$.

Unmethylated CpG motifs are commonly observed in bacterial and viral DNA. Therefore, TLR9 has the potential to recognize unmethylated $\mathrm{CPG}$ motifs in the therapeutic expression cassettes packaged in an AAV capsid and induce innate and adaptive immunity (15). Previous reports revealed that innate immune recognition of the serotype AAV2 by plasmacytoid dendritic cells was mediated by TLR9 and dependent on MyD88 signaling, independent of the form of transgene (16). This innate immune sensor has been implicated in promoting immune responses following self- complementary AAV gene transfer, but the underlying mechanisms have not been fully elucidated $(15,17)$.

In this study, we sought to determine the requirement for TLR9 in promoting immune responses following skeletal muscle AAV gene transfer. We compared responses from WT recipients with responses from TLR9 mice that received vectors based on the immunogenic capsid from AAVrh32.33 (18). AAVrh32.33 vectors stimulate a robust transgene and capsid $T$ cell response that results in detectable loss of transgene expression. These findings were translated into an $A A V r h 32.33$ vector with a $C P G$-depleted genome and mice were assayed for immunoreactivity and transgene stability. We report TLR9-dependent initiation of innate and adaptive immune responses toward AAV-associated antigens following skeletal gene transfer. We further demonstrate that CpGdepleted genomes packaged with immunogenic AAV capsids evade the adaptive immune response and establish prolonged transgene expression, showing that the outcome of AAV gene therapy is a function of the inherent immunogenicity of both the capsid and transgene DNA. 


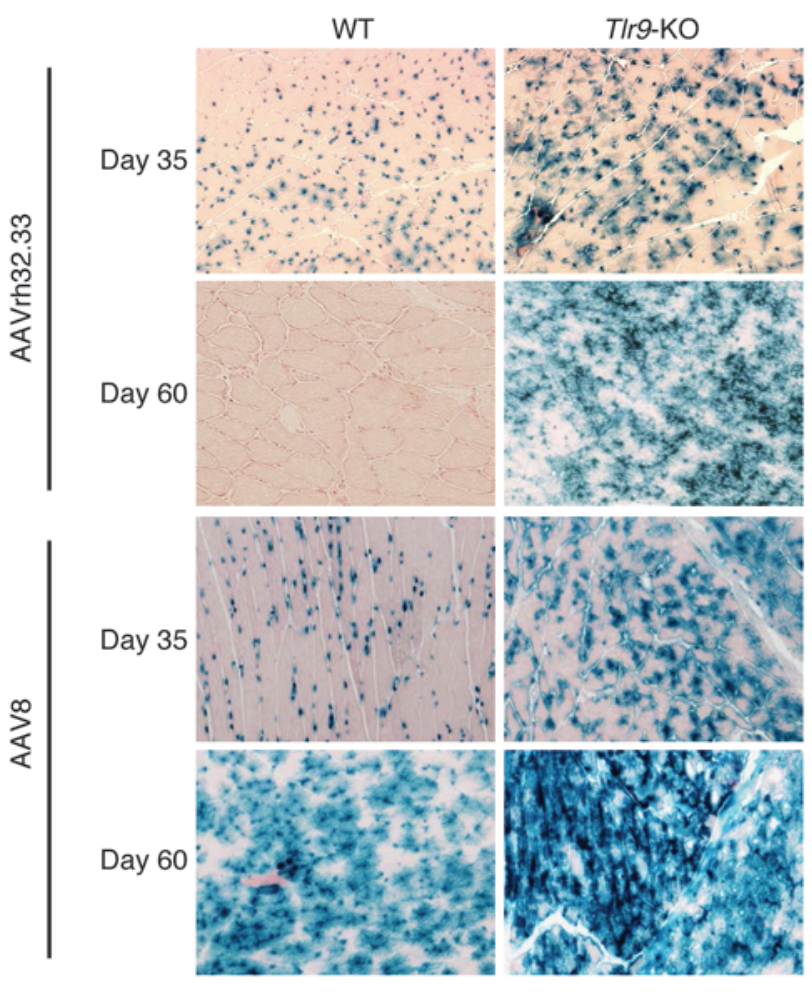

\section{Results}

Studies in C57BL/6 mice demonstrate that intramuscular gene transfer with AAVrh32.33 vectors induces a robust adaptive immune response toward both capsid and transgene antigen, heavy cellular infiltrate, and a loss of detectable transgene expression at days 35 and 60 after administration (18). To evaluate the role of TLR9 signaling in the induction of this adaptive immune response and transgene loss, WT and Tlr9-deficient mice were i.m. injected with $1 \times 10^{11}$ viral particles of AAVrh32.33 expressing a nuclear-targeted $\beta$-gal (nLacZ) reporter gene under

\section{Figure 1}

LacZ expression in skeletal muscle of WT and TIr9-KO mice following AAV gene transfer. X-gal histochemical stain of muscle from WT and TIr9-KO mice injected i.m. with $1 \times 10^{11} \mathrm{GC}$ of AAVrh32.33nLacZ (nuclear LacZ) (top 4 panels) and AAV8nlacZ (bottom 4 panels). Representative sections are shown. $n=4$ mice per group. Original magnification, $\times 10$.

the direction of a chicken $\beta$-actin promoter. Gastrocnemius tissue was recovered at day 35 and day 60 after injection from the WT and Tlr9-KO mice and $\beta$-gal expression in the muscle was assessed by X-gal histochemical stain (Figure 1). WT controls exhibited a complete loss of $\beta$-gal-positive cells at 60 days after injection. In contrast, abrogation of TLR9 signaling resulted in stable transgene expression. These results suggest that TLR9 signaling is required for transgene loss following AAVrh32.33 muscle gene transfer.

To investigate the relationship between TLR9 signaling and immunoreactivity, MHC I tetramer stain and ELISPOT assays were used to quantify transgene reactive $\mathrm{CD}^{+} \mathrm{T}$ cells and primed transgene and capsid-responsive IFN- $\gamma$-producing cells (Figure 2). Peripheral blood cells isolated from whole blood were costained with a FITC-conjugated anti-CD8 $\mathrm{Abs}$ and a PE-conjugated H-2K $\mathrm{K}^{\mathrm{b}}$-ICPMYARV dominant epitope tetramer to determine the percentage of $\mathrm{nLacZ}$-reactive $\mathrm{CD} 8^{+} \mathrm{T}$ cells in the total $\mathrm{CD}^{+} \mathrm{T}$ cell population (Figure 2A). Tlr9-deficient mice exhibited a significant $(P \leq 0.05)$ reduction in the percentage of nLacZ-responsive $\mathrm{CD}^{+} \mathrm{T}$ cell population compared with WT mice. To determine whether TLR9 signaling regulates AAVrh32.33nLacZ cellular immune responses, we employed ELISPOT to quantify the number of in vivo primed capsid and transgene reactive $T$ cells that can respond with IFN- $\gamma$ secretion (Figure $2 B$ ). Elevated capsid and transgene reactive responses were observed in WT but not Tlr9-KO vector recipients, indicating an abrogation of IFN- $\gamma$ secretion in the absence of TLR9 signaling. These findings demonstrate that blocking TLR9 signaling has an inhibitory effect on T cell effector function following stimulation with AAVrh32.33 capsid and transgene antigens.
A

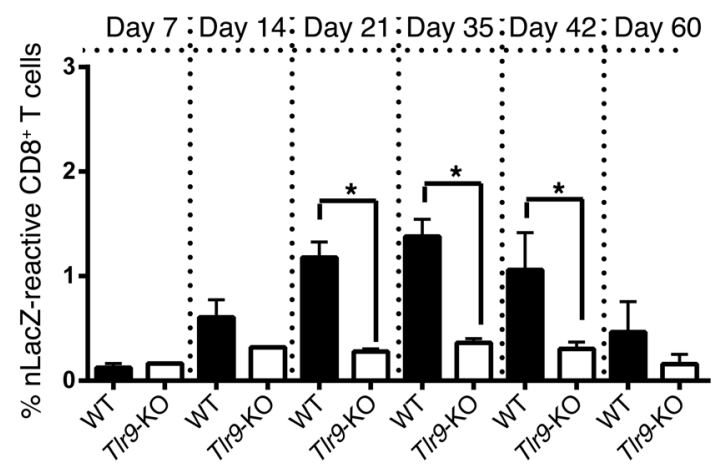

B

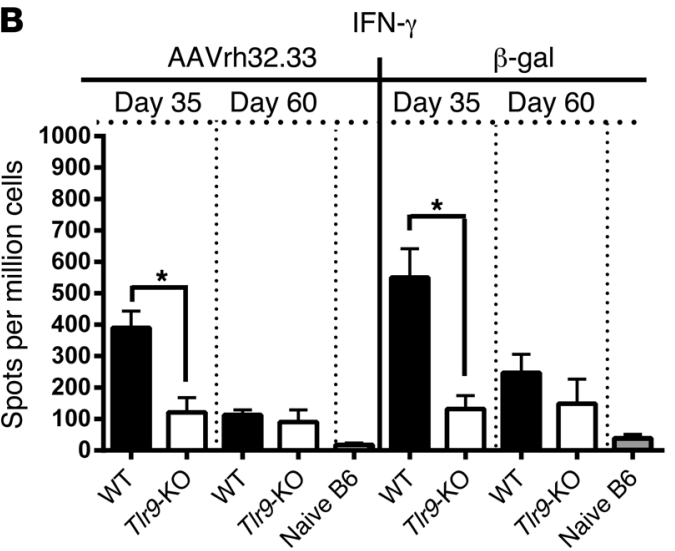

\section{Figure 2}

TIr9 deficiency significantly reduces the percentage of transgene reactive CD8 ${ }^{+} \mathrm{T}$ cells and the Th1 response toward both vector and transgene antigen. WT mice or TIr9-KO mice received i.m. injection of $1 \times 10^{11} \mathrm{GC}$ of AAVrh32.33nLacZ. (A) Lymphocytes isolated from whole blood were stained using the PE-conjugated $\mathrm{H}-2 \mathrm{~K}^{\mathrm{b}}-\mathrm{ICPMYARV}$ tetramer together with FITC-conjugated anti-CD8 Abs to determine the percentage of nLacZ-specific CD8 ${ }^{+} T$ cells in the total CD8 ${ }^{+} T$ cell population. (B) Splenocytes were harvested and processed for ELISPOT assays to quantify primed $\mathrm{CD}^{+} \mathrm{AAVrh} 32.33$ capsid and $\mathrm{nLacZ} \mathrm{T}$ cell immunodominant peptides. Results represent the mean $\pm \mathrm{SD}$ of tetramer positive or cytokineproducing cells from at least $n=3$ recipients per group. ${ }^{*} P<0.05$. 
A
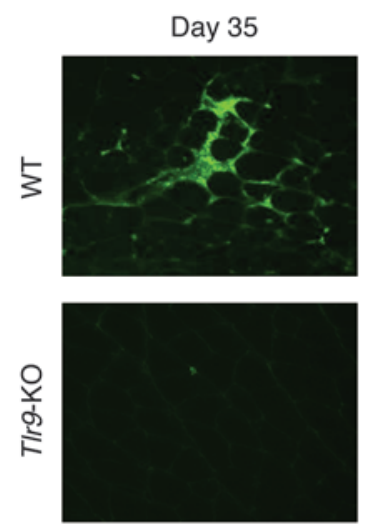

$\mathrm{CD}^{+}{ }^{+} \mathrm{T}$ cells
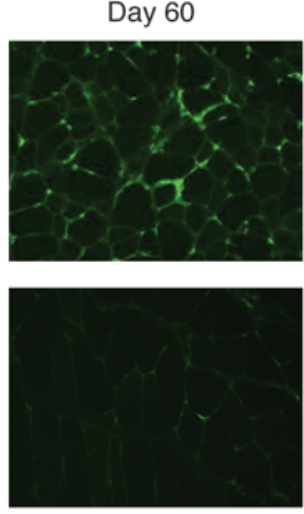

CD8 ${ }^{+} \mathrm{T}$ cells

Day 35
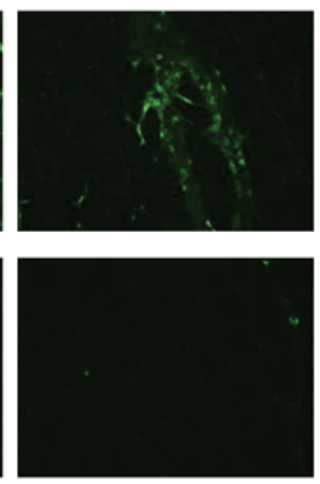

Day 60
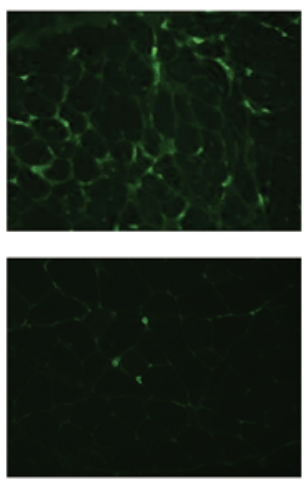

B
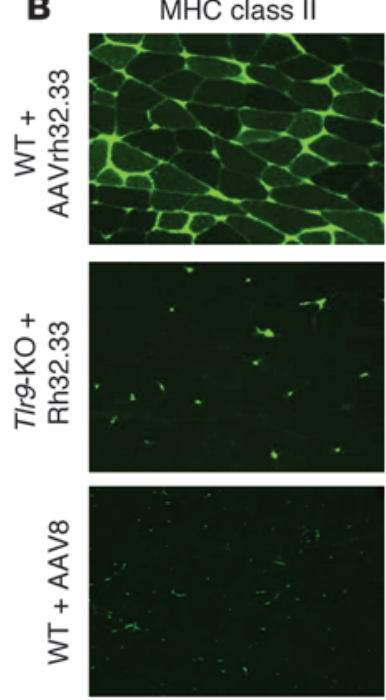

Figure 3

TIr9 deficiency corresponds with minimal cellular infiltrate and blunted MHC class II expression in muscle following AAVrh32.33nLacZ i.m. injection. Muscle section was recovered from WT and TIr9-KO mice 35 and 60 days after i.m. injection of $1 \times 10^{11} \mathrm{GC}$ of AAVrh32.33nLacZ. (A) Sections were stained with anti-CD4 and anti-CD8 Abs and examined by fluorescent microscopy. (B) Muscle section was recovered from WT and TIr9-KO mice after i.m. injection of $1 \times 10^{11} \mathrm{GC}$ of AAVrh32.33nLacZ or AAV8 vectors. Day 35 samples were stained with anti-MHCII Abs. Representative sections are shown. $n=4$ mice per group. Original magnification, $\times 10$.

Heavy $\mathrm{CD}^{+}$and $\mathrm{CD}^{+}$cellular infiltrate has been observed in WT C57BL/6 mice following AAVrh32.33 intramuscular vector transduction (18). To determine the requirement for TLR9 signaling in the induction of this extensive infiltrate, WT and Tlr9-KO muscle cryosections were stained with anti-CD4 and anti-CD8 Abs and examined by fluorescent microscopy at 35 and 60 days after vector administration (Figure 3A). A substantial cellular infiltrate was detected in WT mice. In contrast, minimal cellular infiltrate was observed in $\operatorname{Tl} 9$ - $\mathrm{KO}$ mice. These results are consistent with a TLR9-dependent mechanism of cellular infiltrate in response to intramuscular AAVrh32.33nLacZ transduction.

Historically, AAV8 gene delivery to WT C57BL/6 muscle tissue results in minimal Th1 responses, negligible cellular infiltrate and prolonged transgene expression (18). To investigate the role of TLR9 detection of AAV8 and its effect, if any, on AAV8 gene expression, WT and Tlr9-KO mice were injected i.m. with $1 \times 10^{11}$ genome copies (GC) of AAV8nLacZ. X-gal histochemical stain of muscle sections 35 and 60 days after vector administration was examined (Figure 1). A modest enhancement of expression was observed in the muscle of Tlr9-KO, mice indicating the detection of AAV8 through this innate immune sensor and an effect on transgene expression even in the case of an AAV vector that induces a minimal immune response.

To help delineate the mechanism by which the TLR9-dependent response to $\mathrm{AAVrh} 32.33$ vector extinguishes transgene expression, we evaluated muscle for GC. WT animals dosed with 1011 GC of AAV8 or AAVrh32.33 vectors were sacrificed on day 60 and muscle quantified for total GC, which showed no difference (mean $\pm 1 \mathrm{SD}$, $n=3$; AAV8, $6.6 \pm 1.9 \mathrm{GC} / \mu \mathrm{g}$; and AAVrh32.33 6.3 \pm 2.0 ). This suggests that the adaptive immune response to the immunogenic AAV rh32.33 vector extinguishes expression at a transcriptional or posttranscriptional level.

Muscle tissue possesses a unique function as a nonprofessional APC that can effectively stimulate both $\mathrm{CD}^{+}$and $\mathrm{CD}^{+} \mathrm{T}$ cells to survive, proliferate, and acquire effector function $(13,14)$. To assess the ability of AAVrh32.33 and AAV8 to induce MHC II expression on skeletal muscle, gastrocnemius cryosections from WT and Tlr9-KO mice that received i.m. injections of $1 \times 10^{11}$ GC of AAV8rh32.33nLacZ or AAV8nLacZ were stained with an anti-MHC II Abs and examined by fluorescent microscopy 35 days after gene transfer (Figure 3B). WT muscle tissue transduced with AAVrh32.33nLacZ revealed substantial upregulation of MHC II on skeletal muscle, which was not present on muscle tissue from Tlr9-deficient mice administered AAVrh32.33nLacZ or WT mice that received AAV8. These findings reveal the requirement of TLR9 signaling for MHC II expression on muscle following AAVrh32.33, while CpG present in the vector genomes (VGs) was not sufficient to elicit responses in the context of nonimmunogenic vectors such as AAV8.

Cells infected with viruses induce the expression of numerous chemokines and inflammatory cytokines (19). To assay for innate immune gene transcript induction following intramuscular injection of AAVrh32.33 and AAV8 in WT or Tlr9-deficient mice, we performed quantitative RT-PCR within the first 24 hours after gene transfer to detect innate chemokine and inflammatory cytokine transcript levels (Figure 4). Transcript levels of IL-1 $\beta$, IL-6, MIP-2, and MCP-1 were quantified and expressed as fold induction over mock-treated (PBS injected) WT mice. A dramatic induction of both chemokine and cytokine transcripts was observed in WT mice transduced with AAVrh32.33, while a more moderate expression level was observed in Tlr9-KO mice or in WT mice administered AAV8. Collectively, our data suggest that TLR9 signaling is necessary to induce innate (Figure 4) and adaptive (Figure 2) immune responses toward an immunogenic AAV vector. Our observation that Tlr9-deficient AAVrh32.33 vector recipients exhibited minimal immunoreactivity and stable transgene expression led us to hypothesize that CpG-depleted AAVrh32.33 vectors would escape immune detection and exhibit long-term transgene expression. 

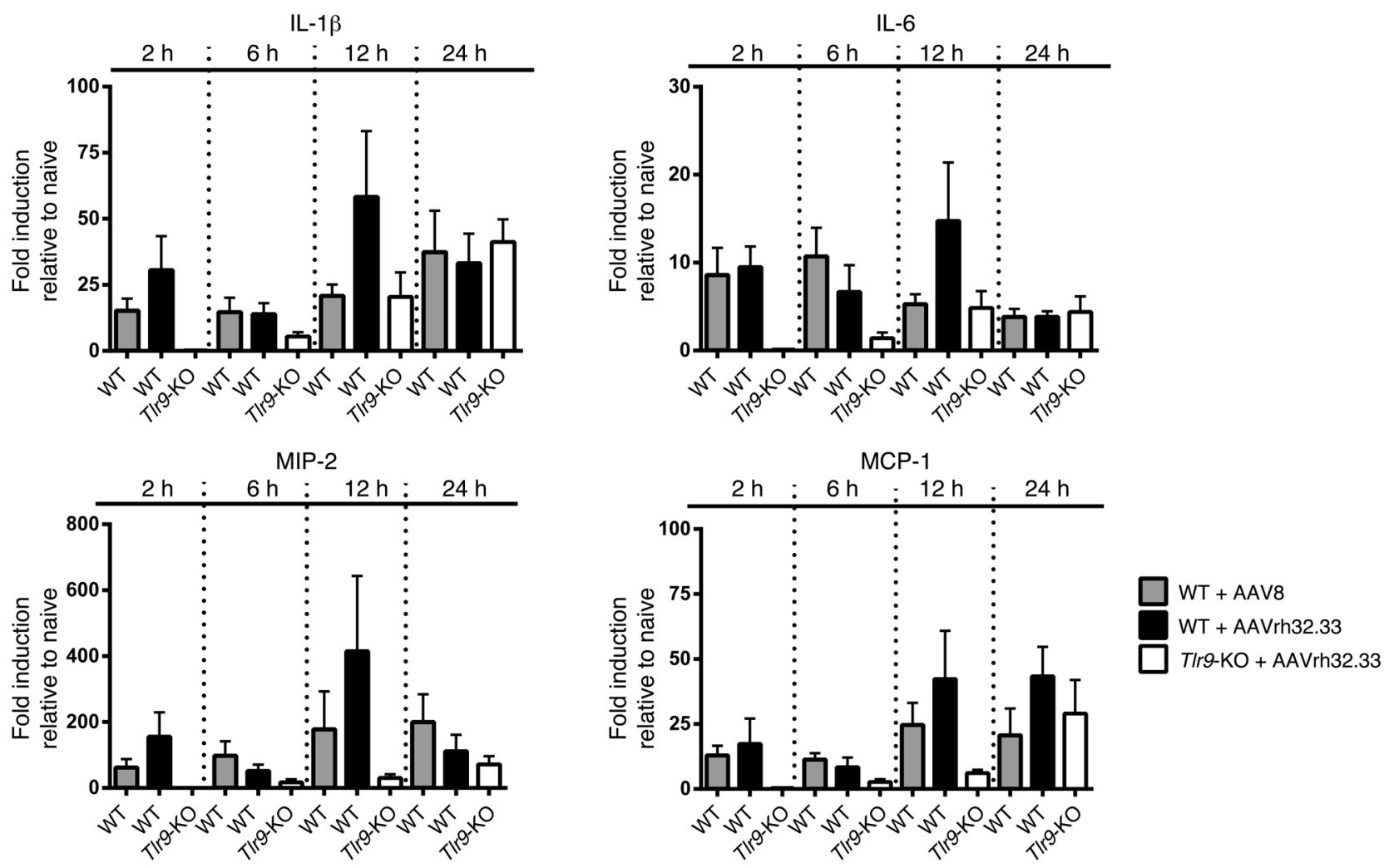

Figure 4

Reduced chemokine and cytokine expression were observed in WT mice transduced with AAV8 and in T/r9-KO transduced with AAVrh32.33 compared with WT mice that received AAVrh32.33. RNA was isolated from the gastrocnemius muscle of WT and TIr9-KO mice administered $1 \times 10^{11}$ GC AAVrh32.33nLacZ or AAV8nLacZ at kinetic time points. Transcript levels of IL-1 $\beta$, IL-6, MIP-2, and MCP-1 were assessed by quantitative RT-PCR. Results depict the mean of RNA expression. $n=8$ mice per group from 2 independent experiments.

Systemic delivery of cationic lipid-plasmid DNA (pDNA)vectors that contain $\mathrm{CPG}$ motifs stimulates acute inflammatory responses with adverse effects on transgene expression (20). CpGdepleted plasmid DNA vectors, on the other hand, exhibit longterm expression and enhanced safety in these kinds of models. To determine whether CPG-depleted AAVrh32.33 vectors would abrogate the robust cellular immune response and transgene loss we observed in the previous experiments (Figures 1-3), we compared the performance of 2 vectors that differed only by the abundance of $\mathrm{CPG}$ motifs. Both vectors contained a cytoplasmic lacZ open reading frame expressed from a mammalian-derived promoter flanked by AAV2 inverted terminal repeats (ITRs). The $\mathrm{CpG}^{+}$vector contained $16 \mathrm{CpGs}$ in the ITRs and $308 \mathrm{CpGs}$ in the lacZ gene and a total of $324 \mathrm{CpGs}$. The $\mathrm{CpG}$ vector was void of CpGs in the lacZ gene, meaning its total CpG content was 16 . CpG-depleted plasmid packaging was up to $80 \%$ as efficient as WT CpG-containing plasmids (data not shown). To assay expression levels, HeLa cells were transfected with $\mathrm{CpG}^{+}$and $\mathrm{CpG}^{-} \mathrm{AAV}$ expression plasmids. Both plasmids express at a quantitatively comparable level (Figure 5A).

To test our hypothesis that CpG-depleted AAVrh32.33 vectors would exhibit prolonged transgene expression, gastrocnemius tissues from WT mice injected i.m. with $1 \times 10^{11} \mathrm{GC}$ of $\mathrm{AAVrh} 32.33 \mathrm{CpG}^{+}\left(\mathrm{RhCpG}^{+}\right)$or AAVrh32.33CpG- $\left(\mathrm{RhCpG}^{-}\right)$ expressing a cytoplasmic $\beta$-gal protein were stained with $\mathrm{X}$-gal (Figure 5, B-E). RhCpG ${ }^{+}$-transduced muscle exhibited a progres- sive loss of detectable $\beta$-gal expression, while the muscle sections from CpG-depleted AAVrh32.33LacZ-transduced mice displayed robust and stable transgene expression. Hence, the steady loss of LacZ transgene expression following AAVrh32.33LacZ gene transfer is dependent on VG CPG motifs consistent with the role of TLR9 activation of innate immunity.

Transgene stability observed in the RhCpG-transduced muscle sections strongly suggests an abrogated adaptive immune response toward transgene and capsid antigen. To assess the requirement for $\mathrm{CPG}$ motifs in the induction of an adaptive immune response toward AAVrh32.33 in the modified vectors, MHC I tetramer stain and ELISPOT assays were used to quantify transgene-reactive $\mathrm{CD} 8^{+}$ $\mathrm{T}$ cells and primed transgene and capsid responsive IFN- $\gamma$-producing $T$ cells as described above (Figure 5, F and G). Mice that received the CPG-depleted AAVrh32.33LacZ vector exhibited a significant reduction $(P \leq 0.05)$ in the percentage of LacZ-responsive $\mathrm{CD}^{+} \mathrm{T}$ cells compared with mice that received the $\mathrm{CpG}^{+}$vector (Figure $5 \mathrm{~F}$ ). Further, a significant decrease of primed transgene and capsid antigen-reactive IFN- $\gamma$ ELISPOT responses was observed in mice that received the $\mathrm{RhCpG}^{-}$but not $\mathrm{RhCpG}^{+}$vector (Figure $5 \mathrm{G}$ ). These findings support the ability of a CpG-depleted vector to escape immunoreactivity following gene transfer.

Minimal cellular infiltrate and MHC II expression were revealed in muscle sections in the absence of TLR9 signaling following AAVrh32.33nLacZ gene transfer (Figure 3, A and B). These data are consistent with a TLR9-dependent mecha- 
A

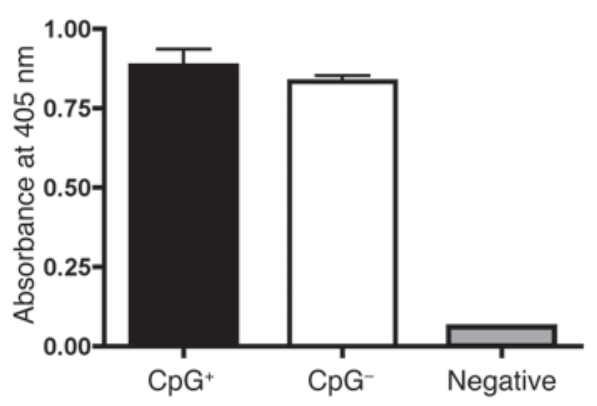

$\mathbf{F}$
LacZ plasmid expression

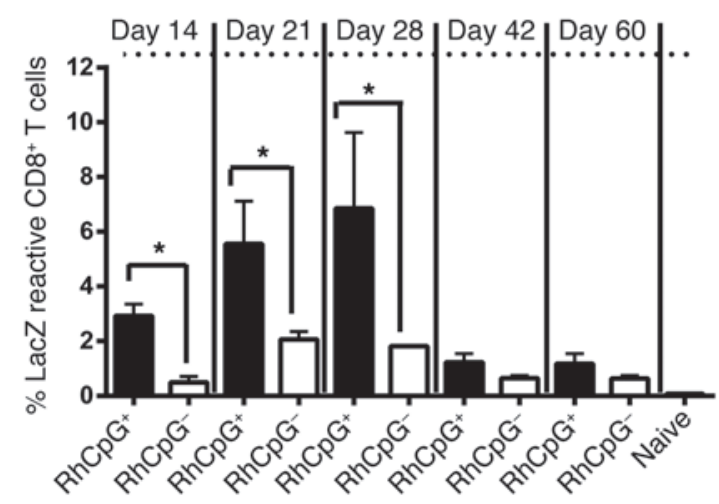

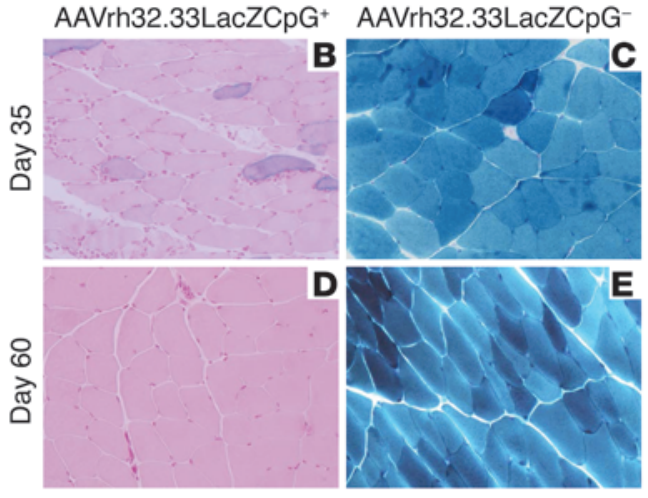

G
IFN- $\gamma$ secretion

AAVrh32.33 $\quad \beta$-gal

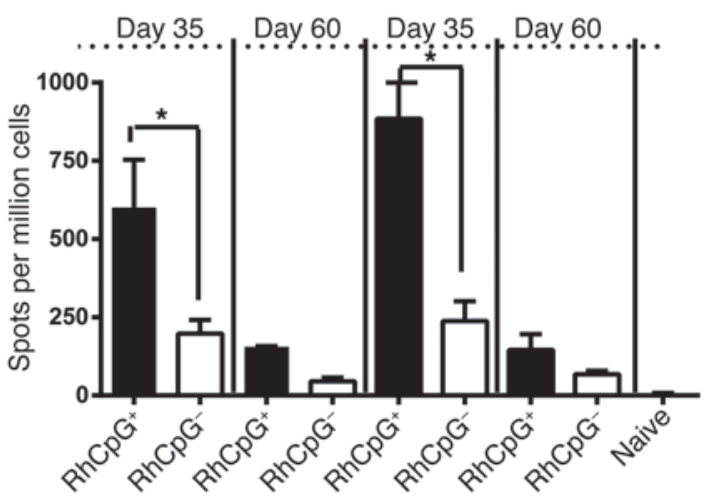

\section{Figure 5}

Biology of CpG-depleted AAVrh32.33LacZ in skeletal muscle. (A) HeLa cells were transfected with $\mathrm{CpG}^{+}$and $\mathrm{CpG}^{-} \mathrm{AAV}$ expression plasmids. Four days after transfection, cells were assayed for $\beta$-gal activity using the Mammalian $\beta$-Galactosidase Assay Kit as instructed for adherent cells. Absorbance was measured at $405 \mathrm{~nm}$ on a TECAN Infinite M1000 PRO plate reader. WT mice were injected i.m. with $1 \times 10^{11} \mathrm{GC}$ of RhCpG+ or $\mathrm{CpG}^{-}$vectors and muscle harvested on day $35(\mathbf{B}$ and $\mathbf{C})$ and day $60(\mathbf{D}$ and $\mathbf{E})$ and stained for X-gal. Representative sections are shown. $n=7$ mice per group. Original magnification, $\times 10$. (F) Lymphocytes were isolated form whole blood and subsequently stained using the PE-conjugated $\mathrm{H}-2 \mathrm{~K}^{\mathrm{b}}-\mathrm{ICPMYARV}$ tetramer together with FITC-conjugated anti-CD8 Abs to determine the percentage of LacZ-specific CD8 ${ }^{+}$T cells in the total $\mathrm{CD}^{+} \mathrm{T}$ cell population. Results represent the mean $\pm \mathrm{SD}$ of tetramer-positive cells from $n=4$ recipients per group. ${ }^{\star} P<0.05$. (G) Splenocytes were harvested and processed for ELISPOT assays to quantify primed CD8 ${ }^{+}$AAVrh32.33 capsid and LacZ T cell immunodominant peptides. Results represent the mean $\pm \mathrm{SD}$ of $\mathrm{CD} 8^{+}$LacZ-reactive T cells or cytokine-producing cells from at least $n=7$ recipients per group. ${ }^{*} P<0.05$.

nism of cellular infiltrate and MHC II skeletal muscle gene induction in response to the immunogenic AAV vector. If TLR9 signaling is necessary for these phenomenon, it is reasonable to suggest that CPG-depleted AAVrh32.33LacZ vectors in WT mice should exhibit histological findings similar to those of Tlr9-KO mice. To test this hypothesis, $1 \times 10^{11} \mathrm{GC}$ of $\mathrm{RhCpG}^{+}$and $\mathrm{RhCpG}^{-}$vectors were injected i.m., and muscle sections were stained with anti-CD4, anti-CD8, and anti-MHC II Abs (Figure 6). Consistent with our hypothesis, muscle transduced with AAVrh32.33CpG vector revealed minimal cellular infiltrate and MHC II expression compared with $\mathrm{RhCpG}^{+}$-transduced muscle. These data reveal the ability of $\mathrm{CPG}$-depleted $\mathrm{AAV}$ vectors to establish long-term transgene expression, evade immune activation (Figure 5), prevent the infiltration of effector T cells, and subvert the induction of skeletal muscle MHC II expression (Figure 6).

\section{Discussion}

Despite substantial progress in the field of gene therapy, there are some scenarios whereby immunity toward AAV capsid and transgene antigen may compromise efficacy and lead to toxicity. Here, we elucidate the mechanism by which skeletal muscle senses an immunogenic AAV vector, leading to the design of a CpG-depleted AAV vector that alters the target cell's recognition of the vector, leading to long-term transgene expression and reduced immunity toward AAV-associated antigens. Our data suggest that TLR9 signaling is necessary to induce an innate and adaptive immune response toward AAV-associated antigens. CpG depletion of AAV vectors attenuates AAV-associated T cell responses, inhibits intramuscular MHC II expression, suppresses cellular infiltrate, and promotes transgene stability. In this study, we have demonstrated that CPG-depleted AAV vectors provide a strategy for diminishing AAV-associated immunity and transient transgene expression.

TLR9 has been implicated as a mediator of immunoreactivity toward single-stranded and self-complementary AAVs (scAAVs) due to its ability to recognize unmethylated CPG motifs present in the therapeutic expression cassettes packaged in an AAV capsid (15). Zhu et al. demonstrated the role of TLR9/MyD88 signaling in mouse plasmacytoid dendritic cells as an important mediator of 


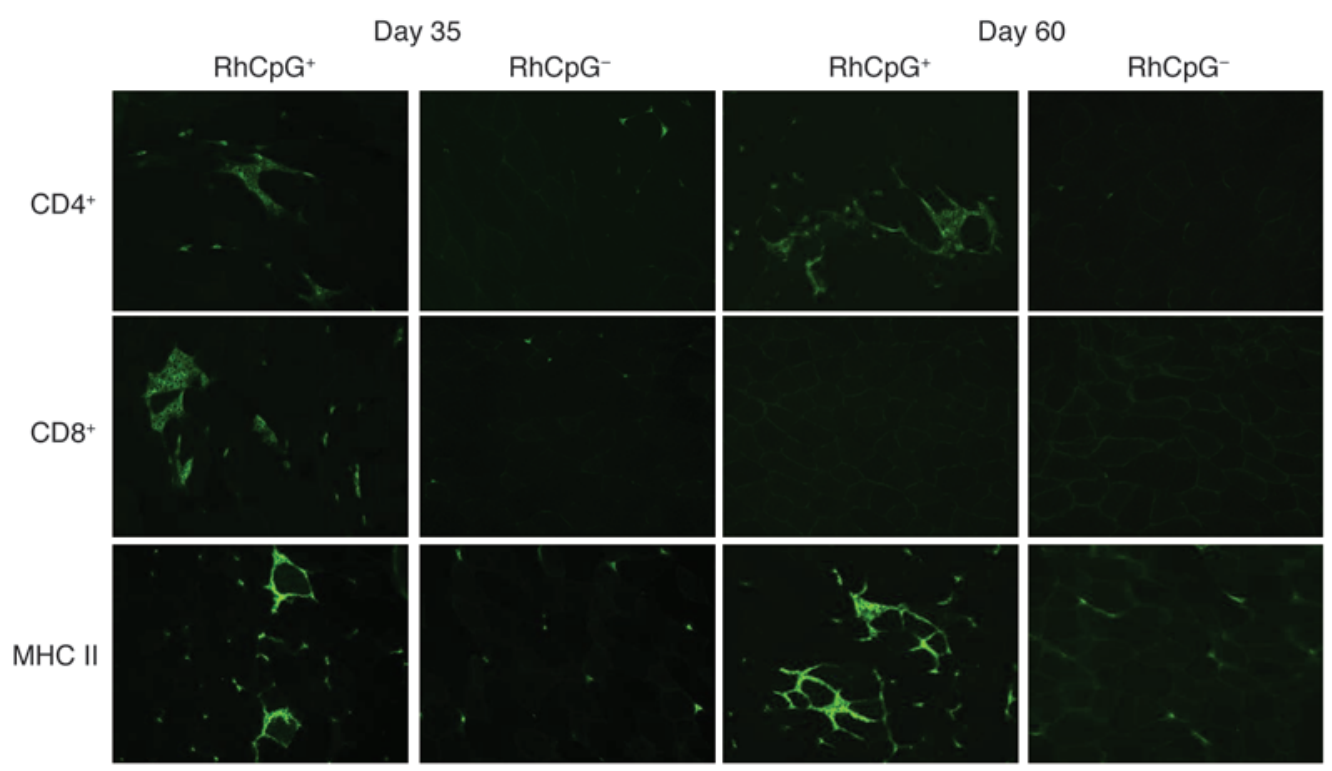

Figure 6

CpG-depleted AAVrh32.33LacZ vector transduction corresponds with minimal cellular infiltrate and $\mathrm{MHC}$ II expression. Muscle section was harvested from WT mice that received i.m. injection of $1 \times 10^{11} \mathrm{GC}$ of $\mathrm{RhCpG}^{+}$ or $\mathrm{CpG}^{-}$vector. Sections were stained with anti-CD4, anti-CD8, and anti-MHC II Abs and examined by fluorescent microscopy. Representative sections are shown. $n=7$ mice per group. Original magnification, $\times 10$. of this approach by evading TLR9 stimulation and thereby reducing inflammation associated with viral transduction. Compared with unmodified vectors, CpG-depleted AAV vectors result in attenuated adaptive immunity, minimal infiltrate, and sustained even increasing - transgene expression over time.

Intramuscular gene transfer has proven to be especially challenging due to adaptive immune responses toward AAV-associated antigens. These findings suggest the predisposition for an immunogenic environment within the skeletal muscle. Direct transduction of APCs by AAV vectors as well as activation through cross-priming mechanisms and antigen transfer from myocytes to APCs undoubt-

the adaptive immune response toward AAV (16). Additional studies examined the effect of TLR9 activation in response to scAAV vector gene transfer and demonstrated increased inflammatory responses toward the double-stranded DNA AAV vector $(15,17)$. In agreement, our data demonstrate that AAVrh32.33 skeletal muscle gene transfer is associated with a robust Th1 response and transgene loss and that TLR9 signaling is necessary for this immune response.

In order to study the role of TLR9 signaling in destructive $\mathrm{T}$ cell responses to AAV muscle gene transfer in mice, we were required to use a capsid that is uniquely immunogenic in mice. This capsid was isolated from rhesus macaques and was shown to elicit strong CTL responses to antigenic transgene products unlike that seen with any other AAV capsids. We showed that the driver for these adoptive responses was AAVrh32.33 stimulation of the innate immune response, as evidenced by the production of proinflammatory cytokines, including IL-1 $\beta$ and IL-6, as well as the chemokines MIP-1 and MCP-1. Costimulatory molecules, such as CD40, CD80, and CD86, are also induced on innate immune cells in response to TLR9 stimulation and function to activate antigen-specific $T$ and $B$ cells. In this respect, TLR9 signaling is indirectly capable of mediating an adaptive immune response toward AAVrh32.33-associated antigens, and this is accomplished through the initiation of a cascade of events leading to activation of capsid and transgene-reactive $\mathrm{T}$ cells, which can limit transgene expression. In experimental models, prior approaches to combating the recognition of AAV vectors by TLR9 include pharmacological administration of oligonucleotide inhibitors at the time of vector transduction (15). Two complications arising from this therapy are the uncertainty of the quantity of antagonist required for therapeutic effect and the length of time the inhibitor would need to be administered. CPG-depleted AAV vectors overcome the limitations edly contribute $(21,22)$. A more controversial contribution may reside in the ability of myocytes to act as facultative APCs (14). Investigations employing CpG-containing DNA vaccines have revealed the contribution of unmethylated $\mathrm{CPG}$ motifs to induce the expression of chemokines, MHC class II molecules, and the costimulatory molecule BB-1 on myocytes (13). The induction of chemokines by myocytes recruits inflammatory cells secreting IFN- $\gamma$, a cytokine characterized as upregulating myocyte MHC II expression and costimulatory ligands. Activated myocytes are subsequently capable of presenting plasmidencoded antigens to stimulate $\mathrm{CD}^{+} \mathrm{T}$ cells to initiate, amplify, and sustain inflammatory responses to DNA vaccines in vitro $(13,14)$. AAVrh32.33 gene transfer robustly induces MHC II expression on transduced myocytes, and this induction is dependent on TLR9 signaling. Our results indicate that skeletal muscle cells transduced with AAVrh32.33 vectors may not simply act as generators of transgene protein but potentially could play an active role in presenting AAV-associated peptides to $\mathrm{CD}^{+} \mathrm{T}$ cells, resulting in enhanced immunogenicity unless AAVrh32.33 vectors are $\mathrm{C} \mathrm{pG}$ depleted.

In contrast with AAVrh32.33 gene transfer, AAV8 gene delivery to WT skeletal muscle results in minimal innate and adaptive immune responses, negligible cellular infiltrate, and prolonged transgene expression, suggesting the inability of AAV8 to robustly activate TLR9 signaling (18). Interestingly, slightly enhanced transgene expression was detected in the skeletal muscle of the Tlr9-deficient mice, indicating the detection of AAV8 through this innate immune sensor and an influence on transgene expression. However, this effect was far less dramatic than what ings suggest differential activation of TLR9 following AAV8 or AAVrh32.33 transduction. The kinetics of uncoating are distinct between serotypes, and it is rational to hypothesize that the capwas observed following AAVrh32.33 gene transfer. These find- 
sids of various AAV serotypes may differ in fragility and uncoat at a higher rate within the endosome, thereby exposing their VGs to TLR9. These findings also indicate that CPG depletion of additional AAV serotypes or other DNA gene delivery vectors may enhance transgene expression.

The apparent immune evasion of AAV8 to antigenic transgene products in mouse models is not relevant to larger animals such as primates and dogs (23-25), in which vibrant and destructive CTLs are elicited. In fact, the study of AAVrh32.33 in mice may better reflect the anticipated biology of other AAV capsids in large animals and humans. Our study reveals the critical role of TLR9 signaling in the activation of innate and adaptive immunity toward this more immunogenic AAV vector, AAVrh32.33. In a skeletal muscle gene transfer model, this signaling pathway leads to LacZ-reactive $T$ cell responses, heavy cellular infiltrate, and transgene loss. CpG-depleted vectors circumvent TLR9 activation, resulting in attenuated adaptive immune responses, substantially reduced cellular infiltrate, and prolonged transgene expression. CpG-depleted AAV vectors provide a platform for improvements in safety and efficacy of AAV gene transfer in humans.

\section{Methods}

Mice. C57BL/6 WT mice were ordered from The Jackson Laboratory. Tlr9KO mice were a gift from Phillip Scott (University of Pennsylvania). All mice were housed under specific pathogen-free conditions in the TRL Animal Facility at the University of Pennsylvania.

$A A V$-mediated transduction of the muscle. AAV8 and AAVrh32.33 pseudotyped vectors were produced with a genome composed of AAV2 ITRs flanking a nuclear targeted form of $\beta$-gal (nLacZ) under the transcriptional control of a CMV-enhanced chicken $\beta$-actin (CB) promoter (26). The 2 cytoplasmic lacZ vectors used in this study differed only in the presence (i.e., 308) or absence (i.e., zero; Invitrogen) of $\mathrm{CpG}$ sequences in the lac $Z$ transgene. The only other sequences in both vectors that contained CpGs are the ITRs (16 CpGs); all other sequences shared in both vectors were completely depleted of $\mathrm{CPG}$ sequences, including the human elongation factor $1-\alpha(\mathrm{E} 1 \mathrm{~F}-\alpha)$ promoter, CMV enhancer, intron, SV40 3' UTR, and ITRs. Both $\mathrm{CpG}^{+}$and $\mathrm{CpG}^{-}$vectors were packaged in AAVrh32.33 capsids. AAV vectors were produced by a scaled-down version of a previously described method by triple transfection of VG, AAV helper, and adenovirus helper plasmids (27). Purification of vectors involved a single iodixanol step gradient and subsequent DNAse treatment. Real-time PCR using a primer/probe set corresponding to the poly A region of the vector and linearized plasmid standards determined genome titer $(\mathrm{GC} / \mathrm{ml})$ of AAV vectors. All vectors used in this study passed the endotoxin assay (threshold, $10 \mathrm{EU} / \mathrm{ml}$ ) using QCL-1000 Chromogenic LAL Test Kit (Cambrex Bio Science). Vectors were produced by the Penn Vector Core at the University of Pennsylvania. Mice were injected in the gastrocnemius muscle with $10^{11} \mathrm{VGs}$ of AAV in a $50-\mu l$ volume.

Immunohistochemistry. To examine expression of nuclear and cytoplasmic $\beta$-gal, X-gal staining of snap-frozen liver cryosections was performed according to standard protocols (18). Representative sections from 4 mice per group were imaged by using brightfield microscopy with a $10 \times$ objective. To analyze MHC II expression and $\mathrm{CD}^{+} / \mathrm{CD}^{+}$infiltrating cell types within the liver, immunostaining and fluorescent microscopy were performed on acetone-fixed cryosections stained with rat anti-CD4 and antiCD8 Abs (BD Biosciences - Pharmingen) and anti-MHC II Abs (Biolegend) as previously described (18).

MHC class I tetramer staining. PE-conjugated MHC class I H2-k $\mathrm{k}^{\mathrm{b}}-\mathrm{ICP}-$ MYARV tetramer complex was obtained from Beckman Coulter. At kinetic time points after vector injection, tetramer staining was performed on heparinized whole blood cells isolated by retroorbital bleeds. Cells were costained for 30 minutes at room temperature with PE-conjugated tetramer and FITC-conjugated anti-CD8a (Ly-2) Abs (BD Biosciences - Pharmingen). Red blood cells were lysed, and cells were fixed with iTAg MHC tetramer lysing solution supplemented with fix solution (Beckman Coulter) for 15 minutes at room temperature. The cells were then washed 3 times in PBS and resuspended in $1 \times$ PBS. Data were gathered with an FC500 Flow Cytometer (Beckman Coulter) and were analyzed with FlowJo analysis software (Tree Star Inc.). In the analysis, lymphocytes were selected on the basis of forward and side scatter characteristics, followed by selection of $\mathrm{CD}^{+}$cells, and subsequently, the tetramer-positive $\mathrm{CD}^{+} \mathrm{T}$ cell population.

ELISPOT assays for cytokine-producing cells. T cell medium consisted of the following: DMEM (Cellgro; Mediatech) supplemented with 10\% heatinactivated FBS (Hyclone), 1\% penicillin/streptomycin (Cellgro; Mediatech), 1\% L-glutamine (Cellgro; Mediatech), 10 mM HEPES buffer (Cellgro; Mediatech), $0.1 \mathrm{mM}$ nonessential amino acids (Invitrogen), $2 \mathrm{mM}$ sodium pyruvate (Cellgro; Mediatech), and $10^{-6}$ M 2-ME (Cellgro; Mediatech).

Splenocytes were isolated by mechanical dissociation followed by $\mathrm{rbc}$ lysis via hypotonic shock, resuspended at a concentration of $5 \times 10^{6}$ cells $/ \mathrm{ml}$, and plated at $5 \times 10^{5}$ cells/well in triplicate on 96-well round-bottom plates. ELISPOT assays were performed according to the manufacturer's instructions (BD Biosciences). T cell medium supplemented with $2 \mu \mathrm{g} / \mathrm{ml}$ of $\mathrm{H} 2-\mathrm{k}^{\mathrm{b}}$-restricted $\beta$-gal $\mathrm{CD}^{+} \mathrm{T}$ cell epitope (ICPMYARV) and $\mathrm{H} 2-\mathrm{k}^{\mathrm{b}}-$ restricted AAVrh32.33 capsid epitope (SSYELPYVM) (Mimotopes) was used to stimulate splenocytes. Splenocytes were incubated at $37^{\circ} \mathrm{C}, 5 \%$ $\mathrm{CO}_{2}$, for 18 hours. Spots were visualized by addition of 3-amino-9-ethylcarbazole (AEC) substrate set (BD Biosciences) and counted using the AID ELISPOT reader system (Cell Technology).

$R N A$ isolation and quantitative RT-PCR. Gastrocnemius muscle tissue was homogenized in $1 \mathrm{ml}$ TRIzol (Life Technologies), and RNA was isolated per the manufacturer's protocol. Total RNA $(5 \mu \mathrm{g})$ was reverse transcribed using 10X PCR buffer (Roche), $10 \mathrm{mM}$ dNTP, oligo (dt), M-MLV-RT (all from Invitrogen), and RNAsin (Promega). Products were then cleaned with $1: 1 \mathrm{phenol} / \mathrm{chloroform} /$ isoamyl (25:24:1) and reprecipitated with $7.5 \mathrm{M}$ NH4OAC in pure ethanol overnight at $-80^{\circ} \mathrm{C}$. Real-time PCR was performed on cDNA using a 7500 Real-Time PCR System (Applied Biosystems). Primer binding to DNA was detected by SYBR 2X Master Mix (Applied Biosystems). Relative expression of the gene of interest was expressed as the comparative concentration of the gene product to the GAPDH product. Transcript relative expression of target genes were then expressed as fold induction over mock-treated (PBS injected) naive WT mouse gastrocnemius muscle samples. Significance was determined with an unpaired Student's $t$ test.

$\beta$-Gal assay. HeLa cells were plated at 100,000 cells/well in a 24-well plate the day prior to transfection. On the day of PEI-mediated transfection, each plasmid sample was diluted in DMEM (130 $\mu \mathrm{l}$ DMEM and $3.2 \mu \mathrm{g}$ $\mathrm{CpG}^{+}, \mathrm{CpG}^{-}$, or control plasmid); then $6.4 \mu \mathrm{l} \mathrm{PEI}(1 \mu \mathrm{g} / \mu \mathrm{l})$ was added to diluted DNA and the solution was mixed vigorously. After 12.5 minutes of incubation at room temperature, $25 \mu \mathrm{l}$ of transfection solution was added to each well. Four days after transfection, cells were assayed for $\beta$-gal activity using the Mammalian $\beta$-Galactosidase Assay Kit (Thermo Scientific) as instructed for adherent cells. Absorbance was measured at $405 \mathrm{~nm}$ on a TECAN Infinite M1000 PRO plate reader.

Statistics. Data were analyzed with GraphPad Prism 4.0c software using unpaired Student's $t$ tests. $P \leq 0.05$ was considered statistically significant.

Study approval. All animal procedure protocols were approved by the Institutional Animal Care and Use Committee of the University of Pennsylvania. 


\section{Acknowledgments}

This work was supported by the following grants: ReGenX SRA (to J.M. Wilson), R01 HL091096 (to J.E. Rabinowitz), T32 AI007324 (to S.M. Faust), and T32 HL007954 (to S.M. Faust).

Received for publication December 7, 2012, and accepted in revised form March 28, 2013.

1. Brantly ML, et al. Sustained transgene expression despite $\mathrm{T}$ lymphocyte responses in a clinical trial of rAAV1- AAT gene therapy. Proc Natl Acad Sci U S A. 2009;106(38):16363-16368.

2. Flotte TR, Mueller C. Gene therapy for alpha1 antitrypsin deficiency. Hum Mol Genet. 2011; 20(R1):R87-R92.

3. Flotte TR, et al. Phase 2 clinical trial of a recombinant adeno-associated viral vector expressing alpha1-antitrypsin: interim results. Hum Gene Ther. 2011;22(10):1239-1247.

4. Mendell JR, et al. Sustained alpha-sarcoglycan gene expression after gene transfer in limb-girdle muscular dystrophy, type 2D. Ann Neurol. 2010; 68(5):629-638.

5. Mendell JR, et al. Limb-girdle muscular dystrophy type $2 \mathrm{D}$ gene therapy restores alpha-sarcoglycan and associated proteins. Ann Neurol. 2009; 66(3):290-297.

6. Mendell JR, et al. Dystrophin immunity in Duchenne's muscular dystrophy. $N$ Engl J Med. 2010 . 363(15):1429-1437.

7. Wang Z, Tapscott SJ, Chamberlain JS, Storb R. Immunity and AAV-mediated gene therapy for muscular dystrophies in large animal models and human trials. Front Microbiol. 2011;2:201.

8. Kawai T, Akira S. TLR signaling. Cell Death Differ. 2006;13(5):816-825.

9. Pahl JH, et al. Adenovirus type 35, but not type 5, stimulates NK cell activation via plasmacytoid dendritic cells and TLR9 signaling. Mol Immunol. 2012; 51(1):91-100.

10. Rathinam VA, Fitzgerald KA. Innate immune sens-
Address correspondence to: James M. Wilson, Department of Pathology and Laboratory Medicine, Perelman School of Medicine, University of Pennsylvania, 125 S. 31st Street, Philadelphia, Pennsylvania 19104, USA. Phone: 215.898.0226; Fax: 215.494.5444; E-mail: wilsonjm@mail.med.upenn.edu.

Benjamin J. Cutler's present address is: Washington University in St. Louis, St. Louis, Missouri, USA. ing of DNA viruses. Virology. 2011;411(2):153-162.

11. Akira S, Uematsu S, Takeuchi O. Pathogen recognition and innate immunity. Cell. 2006; 124(4):783-801.

12. Hemmi H, et al. A Toll-like receptor recognizes bacterial DNA. Nature. 2000;408(6813):740-745.

13. Stan AC, Casares S, Brumeanu TD, Klinman DM, Bona CA. CPG motifs of DNA vaccines induce the expression of chemokines and MHC class II molecules on myocytes. Eur J Immunol. 2001; 31(1):301-310.

14. Behrens L, Kerschensteiner M, Misgeld T, Goebels N, Wekerle H, Hohlfeld R. Human muscle cells express a functional costimulatory molecule distinct from B7.1 (CD80) and B7.2 (CD86) in vitro and in inflammatory lesions. J Immunol. 1998; 161(11):5943-5951.

15. Martino AT, et al. The genome of self- complementary adeno-associated viral vectors increases Tolllike receptor 9-dependent innate immune responses in the liver. Blood. 2011;117(24):6459-6468.

16. Zhu J, Huang X, Yang Y. The TLR9-MyD88 pathway is critical for adaptive immune responses to adeno-associated virus gene therapy vectors in mice. J Clin Invest. 2009;119(8):2388-2398.

17. Wu T, et al. Self-complementary AAVs induce more potent transgene product-specific immune responses compared to a single-stranded genome. Mol Ther. 2012;20(3):572-579.

18. Mays LE, et al. Adeno-associated virus capsid structure drives $\mathrm{CD} 4$-dependent $\mathrm{CD}^{+} \mathrm{T}$ cell response to vector encoded proteins. J Immunol. 2009; 182(10):6051-6060.
19. Zaiss AK, Liu Q, Bowen GP, Wong NC, Bartlett JS, Muruve DA. Differential activation of innate immune responses by adenovirus and adeno-associated virus vectors. J Virol. 2002;76(9):4580-4590.

20. Zhao H, Hemmi H, Akira S, Cheng SH, Scheule RK, Yew NS. Contribution of Toll-like receptor 9 signaling to the acute inflammatory response to nonviral vectors. Mol Ther. 2004;9(2):241-248.

21. Ulmer JB, Otten GR. Priming of CTL responses by DNA vaccines: direct transfection of antigen presenting cells versus cross-priming. Dev Biol (Basel). 2000;104:9-14.

22. Fu TM, et al. Priming of cytotoxic T lymphocytes by DNA vaccines: requirement for professional antigen presenting cells and evidence for antigen transfer from myocytes. Mol Med. 1997;3(6):362-371.

23. Bell $P$, et al. Evaluation of adeno- associated viral vectors for liver-directed gene transfer in dogs. Hum Gene Ther. 2011;22(8):985-997.

24. Gao G, et al. Adeno-associated virus- mediated gene transfer to nonhuman primate liver can elicit destructive transgene- specific $\mathrm{T}$ cell responses. Hum Gene Ther. 2009;20(9):930-942.

25. Wang $\mathrm{L}$, et al. The pleiotropic effects of natural AAV infections on liver-directed gene transfer in macaques. Mol Ther. 2010;18(1):126-134.

26. Wang L, Takabe K, Bidlingmaier SM, Ill CR, Verma IM. Sustained correction of bleeding disorder in hemophilia B mice by gene therapy. Proc Natl Acad SciU S A. 1999;96(7):3906-3910.

27. Lock $M$, et al. Rapid, simple, and versatile manufacturing of recombinant adeno-associated viral vectors at scale. Hum Gene Ther. 2010;21(10):1259-1271. 